\title{
Identification of Self-Management Behavior Clusters Among People Living with HIV in China: A Latent Class Profile Analysis
}

\author{
Hong Zhang $\mathbb{D}^{1,2}$ \\ Yao Yin' \\ Huan Wang' \\ Ying $\mathrm{Han}^{3}$ \\ Xia Wang' \\ Yi Liu ${ }^{4}$ \\ Hong Chen'
}

'West China School of Nursing, Sichuan University/West China Hospital, Sichuan University, Chengdu, Sichuan Province, People's Republic of China; ${ }^{2}$ Department of Nursing, Medical School, Hubei Minzu University, Enshi, Hubei Province, People's Republic of China; ${ }^{3}$ Clinical Skills Training Center of West China Hospital, Sichuan University, Chengdu, Sichuan Province, People's Republic of China; ${ }^{4}$ Department of Rheumatology and Immunology, West China Hospital, Sichuan University, Chengdu, Sichuan Province, People's Republic of China
Correspondence: Hong Chen West China School of Nursing, Sichuan University/West China Hospital, Sichuan University, No. 37, Guoxuexiang, Wuhou District, Chengdu, Sichuan Province,

People's Republic of China

Tel +86I 8980601733

Email1366I09878@qq.com

Yi Liu

Department of Rheumatology and Immunology, West China Hospital,

Sichuan University, No. 37, Guoxuexiang,

Wuhou District, Chengdu, Sichuan

Province, People's Republic of China

Tel +861 8980602061

Emailyi2006liu@I63.com
Background: Self-management directly affects the health outcomes and quality of life among people living with HIV (PLWH). A better understanding of self-management level will provide evidence for researchers to develop effective interventions.

Purpose: This study aims to identify the latent classes among PLWH in their levels of selfmanagement behavior, and to explore the sociodemographic and disease-related predictors within these classes.

Materials and Methods: A total of 868 PLWH were recruited from August 2017 to January 2019 in Sichuan Province, China. A latent class profile analysis was used to identify participants' self-management behavior, and multinomial logistic regression was used to explore the sociodemographic and disease-related predictors of the different latent classes.

Results: Model fit indices supported a three-class model. The mean self-management scores in the three classes were $23.56(\mathrm{SD}=6.02), 37.91(\mathrm{SD}=3.80)$, and $47.95(\mathrm{SD}=4.18)$, respectively. The latent classes were Class 1 (a poor level of self-management behavior, $12.1 \%$, $\mathrm{n}=104$ ), Class 2 (a moderate level of self-management behavior, 56.1\%, $\mathrm{n}=491$ ) and Class 3 (a good level of self-management behavior, 31.7\%, n=273). Antiretroviral trerapy (ART) status, infection route, and educational level were the main predictors of self-management behavior.

Conclusion: The findings indicated that the level of self-management behaviors among PLWH in China is inadequate. Those with a lower educational level, who were infected through blood/injecting drugs, and who were not receiving ART, showed a significantly lower level of self-management behavior. These results could help healthcare professionals to quickly recognize PLWH who are at a high risk of low-level self-management, using individual characteristics and could provide a scientific basis for the development of effective and targeted programs to improve self-management level in PLWH.

Keywords: China, cross-sectional study, HIV, AIDS, self-management

\section{Introduction}

HIV is a serious threat to human health and has a significant impact on societal development. ${ }^{1}$ More than 36 million people have been infected worldwide. ${ }^{2}$ There were 664,751 people living with HIV (PLWH) in China by the end of $2016 .{ }^{3}$ With the introduction of antiretroviral therapy (ART) for HIV, the mortality decreased, and the life expectancy of PLWH increased significantly, ${ }^{4}$ AIDS has been transformed into a chronic disease. Effective self-management is critically important for chronic diseases. ${ }^{5}$ Self-management is the gold standard of chronic care. ${ }^{6}$ It has 
been reported to enhance people's confidence in facing their disease, improve their health awareness and social adaptability, promote the development of good habits, ${ }^{7}$ and improve their prognosis, reducing the burden on the medical and healthcare systems. ${ }^{8}$ Adherence to effective selfmanagement is critical for people living with chronic diseases, and could yield important benefits including improvements in quality of life, reductions in the utilization of healthcare resources, ${ }^{9}$ and decreased transmission of HIV. ${ }^{10}$ Better self-management promotes the establishment of a healthy lifestyle and safer sexual behavior, improves medication adherence, reduces anxiety, and improves quality of life in PLWH. ${ }^{1}$

Self-management refers to a person managing their own needs generated by the illness (taking medication, daily physical health activities), activating social support (family, peers, and HIV care providers), and living with chronic illness. ${ }^{11,12}$ Self-management is a central concept of the Individual and Family Self-Management Theory (IFSMT). ${ }^{13}$ IFSMT was developed to describe multiple levels of self-management in chronic disease including HIV. ${ }^{14}$ This middle-range theory is descriptive and comprises dynamic and interrelated constructs defined as the context, the process of self-management, selfmanagement interventions and self-management outcomes. The contextual factors focus on "risk and protective factors", including condition-specific factors, the physical and social environment, and individual factors. $^{15}$ In the IFSMT, the risk and protective factors in the context dimension affect the individual's and family's ability to engage in the process dimension of selfmanagement, and have a direct impact on outcomes. ${ }^{14}$ Individual characteristics are also viewed as responsible for enhancing or inhibiting engagement in selfmanagement behaviors. Individual factors, including age, gender, educational level, and income, may affect individual's self-management behaviors. ${ }^{16,17}$

Previous studies demonstrate variable levels of selfmanagement among PLWH across different countries, ${ }^{16,18}$ and there are a few studies of the selfmanagement behavior of PLWH in China. ${ }^{17,19,20}$ However, to the best of our knowledge there is no empirical classification of different levels of self-management behaviors among PLWH. Unarguably, there is a need to understand the subgroups of self-management behaviors and to identify possible predictors of self-management levels. With this information, healthcare providers will be able to quickly identify people at a high risk for low level of self-management, using individual characteristics, and this might help to tailor targeted and appropriate interventions, thus improving self-management in PLWH.

Sichuan Province, located in West China, is characterized by the largest population of PLWH in China with 92,976 HIV/AIDS patients alone by the end of $2016 .^{21}$ Of all the subjects, $34.16 \%$ were in Liangshan Yi Autonomous Prefecture (Liangshan Prefecture), and $20.88 \%$ were in Chengdu, with relatively low numbers (1-2\%) in Panzhihua. ${ }^{21}$ We conducted a cross-sectional study of PLWH in these three areas within Sichuan Province. The aims of this study were (1) to identify the level and the latent classes of self-management behavior and (2) to explore sociodemographic and disease-related predictors of different latent classes.

\section{Materials and Methods Ethical Statement}

All procedures performed in the study involving human participants were in accordance with the ethical standards of the institutional research committee and the guidelines of the Declaration of Helsinki. Ethical approval for the research was obtained from the West China Hospital Medical Ethics Committee (Num: 20170430) before data collection.

\section{Study Design and Sample}

A cross-sectional study was conducted from August 2017 to January 2019. All of the participants were from designated HIV treatment sites, ie, Centers for Disease Control (CDCs) or hospitals, in three areas (Chengdu, Panzhihua, and Liangshan Prefecture). The inclusion criteria for participants were (1) participants met the Chinese Ministry of Health's (2005) diagnostic criteria for HIV, (2) participants were 18 years or older, (3) participants were able to understand and answer the questionnaires, and (4) participants volunteered freely for the survey. Participants with a mental disorder or cognitive impairment were excluded.

\section{Data Collection}

The investigators were trained in the study protocol and data collection. Participants were recruited using convenience sampling when they attended the participating AIDS clinics or CDCs to receive antiretroviral drugs or health counseling. All participants were informed of the purpose, content and potential risks of the study. All participants signed written informed consent forms. Then, they 
completed the paper-based questionnaires anonymously in private rooms. A total of 950 PLWH were recruited. Of these, 50 participants refused to fill out the questionnaires, and 900 questionnaires were completed (a response rate of $94.7 \%$ ), of which 32 were discarded due to a lack of data on major variables. Finally, 868 questionnaires were included for analysis (the effective response rate was $96.44 \%$ ). Nylund-Gibson et $\mathrm{al}^{22}$ suggested that a total of 300 or more cases is desirable for an appropriate sample size in latent class profile analysis. The sample size of this study $(n=868)$ is therefore adequate. Each participant took approximately 25-30 minutes to complete the survey and received $¥ 50$ as labor compensation.

\section{Measures}

Self-management was assessed using the HIV SelfManagement Scale that was developed by Webel. ${ }^{23}$ This scale is a self-reported questionnaire and each domain demonstrated acceptable reliability $(0.72-0.86)$ and stability $(0.61-0.85){ }^{23}$ The scale includes three domains and 20 items: daily self-management health practices (12 items), which represents self-regulation skills and abilities, (eg, dietary adjustments); social support and HIV selfmanagement (three items), which represents the social facilitation aspect, including social influence, social support, and collaboration with healthcare professionals (eg, participation in support groups); and the chronic nature of HIV self-management (five items), which represents knowledge, beliefs, and self-efficacy (eg, AIDS management is a top priority). The total score of the scale was calculated by adding together all the items' scores. Possible scores range from 0-60. A higher score indicates a higher level of self-management. We have obtained Webel's permission to translate and use the scale. After translation, retranslation, cultural adjustment, preinvestigation and analysis, the content validity index of the scale was 0.910 , the index of each item was 0.769 1.000 , the internal reliability of Cronbach's $\alpha$ was 0.943 , the coefficient of each dimension was $0.820-0.959$, and the total test-retest reliability of the scale was 0.965 .

Sociodemographic and clinical data of the participants were collected using a 14-item questionnaire developed for the purposes of the study, based on the IFSMT and variables identified in the literatures. Sociodemographic questions encompassed the individual, physical and social environment, such as region (Chengdu, Panzhihua, and Liangshan), gender, marital status, age, religion, ethnicity, educational level, residence (city/town, countryside), occupation, and per capita monthly household income. Clinical factors influencing the patient's health status included years since HIV diagnosis, infection route, ART status and HIV serostatus disclosure.

\section{Data Analysis}

SPSS 20.0 (IBM Corporation, Armonk, NY, USA) and Mplus Version 7.4 were used for data analysis. SPSS was used for descriptive statistics (frequency, mean, standard deviation), Chi-square test, analysis of variance (ANOVA) and multinomial logistic regression analysis. A Latent Class Profile Analysis(LCPA) was performed using Mplus. LCPA is a person-centered analysis method, used to identify unmeasured class membership among participants, using multiple measured variables, ${ }^{24}$ and is typically used for continuous variables. ${ }^{25}$ The HIV selfmanagement scores are continuous data and so LCPA was used to identify latent classes of self-management behavior reported by PLWH. Robust maximum likelihood was selected as the estimation method. ${ }^{26}$ Akaike Information Criterion (AIC), Bayesian Information Criterion (BIC) and Sample Size Adjusted BIC (SABIC) were the main indicators used to evaluate the quality of the different models. The lower the value, the better the model fit. ${ }^{27}$ Relative entropy was used to evaluate the uncertainty of the models, and their values ranged from $0-1$, a higher value indicating greater classification certainty. The likelihoodbased tests, the Vuong-Lo-Mendell-Rubin adjusted likelihood ratio test (VLMR-LRT) and the bootstrapped likelihood ratio test (BLRT), provided $\mathrm{p}$ values assessing whether adding a class led to a statistically significant improvement in model fit. These likelihood-based tests compared the fit between two neighboring class models. A nonsignificant $\mathrm{p}$ value for a $\mathrm{k}$ class solution thus lent support for the k-1 class solution. ${ }^{22}$ Multinomial logistic regression was used to recognize the predictors of different latent subgroups of self-management behavior. The variables of sociodemographic and clinical data with statistical significance $(p<0.05)$ in the Chi-square test were regarded as the independent variables, and the identified potential classes of self-management behavior were the dependent variable in multinomial logistic regression.

\section{Results}

\section{Characteristics of the Participants}

The age of the 868 participants ranged from 18 to 82 years, with a mean age of 43.68 years $(S D=13.90)$. All 
of the minority were of Yi nationality. The majority of participants were receiving ART $(89.7 \%, n=779)$. There are 152 patients infected through the blood/injecting drugs, of which $77.63 \% \quad(n=118)$ were from Liangshan Prefecture, 14.47\% $(n=22)$ from Panzhihua, and 7.89\% $(n=12)$ from Chengdu. Other sociodemographic and clinical data of the participants are presented in Table 1.

\section{Latent Class Profile Analysis of Self-Management Behavior}

Models with one to four latent classes were examined, where the one-class model was set as a baseline. The fitting index summary of LCPA of self-management behavior is presented in Table 2. According to the indicators, a nonsignificant $\mathrm{p}$ value for a four-class model suggests that the three-class model is supported by the VLMR-LRT. Model 3 was the best, compared with the other three models, because of its smaller values of log-likelihood, $\mathrm{AIC}, \mathrm{BIC}$ and SABIC, and the highest entropy value. In addition, VLMR-LRT $(p<0.05)$ and BLMR $(p<0.05)$ for this model both showed statistical significance. After careful review of all the models, the three-class model for selfmanagement behavior was shown to best satisfy the selection criteria. The conditional probability distribution of the three potential categories of PLWH is shown in Figure 1. Self-management behavior was divided into three potential categories, the proportions of the three classes were $12.1 \%$ $(n=104), 56.1 \%(n=491)$, and $31.7 \%(n=273)$ respectively.

\section{The Distribution of the Self-Management Scores Among the Three Classes}

The mean self-management score of all PLWH was 39.35 $(\mathrm{SD}=8.50)$. ANOVA was used to examine the distribution of the self-management scores among the three classes. The effect size was denoted by $\mathrm{\eta}^{2}$. Fisher's least significant difference test (LSD-t) was used to identify significant differences between the classes. The mean selfmanagement scores in the three classes were 23.56 $(\mathrm{SD}=6.02), 37.91(\mathrm{SD}=3.80)$, and $47.95(\mathrm{SD}=4.18)$ respectively, $\mathrm{F}(2,865)=1312.00, \mathrm{y}^{2}=0.752, \mathrm{p}<0.001$. Class 1 compared with Class 2, $\mathrm{t}=-31.41, \mathrm{p}<0.001,95 \%$ CI (15.25, -13.46); Class 1 compared with Class $3, \mathrm{t}=$ -49.97, $\mathrm{p}<0.001,95 \%$ CI $(-25.35,-23.43)$; Class 2 compared with Class 3, $\mathrm{t}=-31.35, \mathrm{p}<0.001,95 \%$ CI $(-10.66$, -9.40). The results indicate that Class 3 had the highest level of overall self-management, whereas Class 1 had the lowest level. We therefore concluded that Class 1 had a poor level of self-management behavior, Class 2 had a moderate level of self-management behavior, and Class 3 had a good level of self-management behavior.

\section{The Distribution of the}

\section{Sociodemographic and Clinical Data Among the Three Classes}

The distribution of the sociodemographic and clinical data among the three classes is presented in Table 3. A Chisquare test was used to examine the three latent classes according to their different sociodemographic and clinical data, and $\mathrm{p}<0.05$ was considered statistically significant. There was a significant difference in the distribution of region, age, ethnicity, educational level, religion, residence, occupation, per capita monthly household income, infection route, ART status, and HIV serostatus disclosure among three classes (all $\mathrm{p}<0.05$ ), but not in the distribution of gender, marital status, or years since HIV diagnosis between the three classes (all $\mathrm{p}>0.05$ ).

\section{Predictors of Three Latent Classes of Self-Management Behavior}

Multinomial logistic regression (with one subgroup as a base scenario) was used to determine whether sociodemographic and clinical data are predictors of subgroup membership. The results are shown in Table 4. The model of Class 3 versus Class 1 indicates that educational level, infection route, and ART status are significant predictors. PLWH who had a higher educational level, who were not infected through blood/injecting drugs, and who were receiving ART, were more likely to report better level of self-management behavior. The Model Class 2 versus Class 1 indicates that infection route and ART status are significant predictors. PLWH who were infected through blood/injecting drugs, and who were not receiving ART were more likely to report lower level of self-management behavior.

\section{Discussion}

Self-management directly affects the health outcome and quality of life in PLWH, and it plays an important role in the management of chronic diseases and prevention of comorbidities. ${ }^{18}$ Self-management is an important component of the long-term disease management in PLWH, and has potential to improve health outcomes of PLWH. ${ }^{28}$ A better understanding of PLWH's level of selfmanagement will help researchers to develop targeted 
Table I Characteristics of the Participants $(n=868)$

\begin{tabular}{|c|c|c|}
\hline Variables & $\mathbf{n}$ & $\%$ \\
\hline \multicolumn{3}{|l|}{ Region } \\
\hline Chengdu & $|8|$ & 20.85 \\
\hline Liangshan & 322 & 37.10 \\
\hline Panzhihua & 365 & 42.05 \\
\hline \multicolumn{3}{|l|}{ Gender } \\
\hline Male & 600 & 69.12 \\
\hline Female & 268 & 30.88 \\
\hline \multicolumn{3}{|l|}{ Age (years) } \\
\hline$\leq 30$ & 149 & 17.17 \\
\hline $31-50$ & 492 & 56.68 \\
\hline$>50$ & 227 & 26.15 \\
\hline \multicolumn{3}{|l|}{ Ethnicity } \\
\hline Han nationality & 552 & 63.59 \\
\hline Minority(Yi people) & 316 & 36.41 \\
\hline \multicolumn{3}{|l|}{ Educational level } \\
\hline Illiterate or Primary school & 420 & 48.39 \\
\hline Junior high school & 208 & 23.96 \\
\hline Senior high school & 108 & 12.44 \\
\hline Junior college or higher & 132 & 15.21 \\
\hline \multicolumn{3}{|l|}{ Marital status } \\
\hline Unmarried & 187 & 21.54 \\
\hline Divorced & 128 & 14.75 \\
\hline Married & 474 & 54.61 \\
\hline Widowed & 79 & 9.10 \\
\hline \multicolumn{3}{|l|}{ Religion } \\
\hline No & 535 & 61.64 \\
\hline Yes & 333 & 38.36 \\
\hline \multicolumn{3}{|l|}{ Residence } \\
\hline City/town & 375 & 43.20 \\
\hline Countryside & 493 & 56.80 \\
\hline \multicolumn{3}{|l|}{ Occupation } \\
\hline Unemployed & 162 & 18.66 \\
\hline Employed & 706 & 81.34 \\
\hline \multicolumn{3}{|l|}{ Per capita monthly household income (yuan) } \\
\hline $0-999$ & 453 & 52.19 \\
\hline $1000-1999$ & 133 & 15.32 \\
\hline 2000-2999 & 173 & 19.93 \\
\hline $3000-$ & 109 & 12.56 \\
\hline \multicolumn{3}{|l|}{ Years since HIV diagnosis } \\
\hline$<1$ & 126 & 14.52 \\
\hline $1-5$ & 441 & 50.81 \\
\hline $5.1-10$ & 248 & 28.57 \\
\hline$>10$ & 53 & 6.11 \\
\hline
\end{tabular}

(Continued)
Table I (Continued).

\begin{tabular}{|l|c|c|}
\hline Variables & $\mathbf{n}$ & $\%$ \\
\hline Infection route & & \\
$\quad$ Unclear & 255 & 29.38 \\
Sexual & $46 I$ & 53.11 \\
Blood/injecting drugs & 152 & 17.51 \\
\hline ART status & & \\
Yes & 779 & 89.75 \\
No & 89 & 10.25 \\
\hline HIV serostatus disclosure & & \\
No & 202 & 23.27 \\
Yes & 666 & 76.73 \\
\hline
\end{tabular}

Abbreviation: ART, antiretroviral therapy.

interventions. This is the first time that LCPA has been applied to the understanding of different behavior classes in self-management among PLWH. The levels of selfmanagement were divided into three latent classes: Class 1 (poor level), Class 2 (moderate level), and Class 3 (good level). Most (68.2\%) participants' level of self-management was poor or moderate. Thus, the level of self-management behaviors in China is inadequate. Health care providers should develope effective interventions to improve patients' self-management behaviors in future.

Based on the Individual and Family Self-Management Theory, ${ }^{14}$ this study examines sociodemographic and clinical variables that impact the level of selfmanagement behaviors in PLWH. Our study shows that educational level is one of the predictors of selfmanagement level. Most (81.1\%) participants with a low level of education (illiterate or primary school) were found to be in classes 1 or 2. This discovery was consistent with previous studies. For example, Efendi et $\mathrm{al}^{29}$ confirm that educational levels significantly influence people's level of knowledge of HIV; selfmanagement scores have been found to be positively related to educational level among PLWH in Northwest Ethiopia; ${ }^{15}$ a higher level of education has been associated with more knowledge about HIV, ${ }^{19}$ which may enhance their self-management behaviors. Thus, PLWH with a better knowledge level tended to be more aware of their health status and better prepared to make decisions about health behaviors. 
Table 2 Fit Indices of LCPA for Self-Management Behavior Clusters $(n=868)$

\begin{tabular}{|l|c|c|c|c|l|l|l|l|}
\hline Model & LL & AIC & BIC & SABIC & Entropy & VLMR-LRT, p & BLRT, p & Mixing Ration \\
\hline 1 & $-20,886.21$ & $41,852.42$ & $42,043.07$ & $41,916.04$ & & & & \\
2 & $-19,771.18$ & $39,664.35$ & $39,955.09$ & $39,761.37$ & 0.84 & 0.001 & $<0.001$ & $0.280,0.720$ \\
3 & $-19,370.21$ & $38,904.41$ & $39,295.24$ & $39,034.83$ & 0.88 & 0.014 & $<0.001$ & $0.121,0.561,0.317$ \\
4 & $-19,098.85$ & $38,403.71$ & $38,894.63$ & $38,567.52$ & 0.85 & 0.334 & $<0.001$ & $0.123,0.367,0.279,0.231$ \\
\hline
\end{tabular}

Abbreviations: LL, the Log-Likelihood; AIC, the Akaike Information Criterion; BIC, the Bayesian Information Criterion; SABIC, the Sample-Size Adjusted BIC; VLMR-LRT, Vuong-Lo-Mendell-Rubin adjusted likelihood ratio test; BLRT, Bootstrapped Likelihood Ratio Test; p, p-value.

ART status was found to be one of the main predictors of self-management level. Self-management in PLWH is critical for implementing and maximizing the benefits of ART. ${ }^{8}$ In this study, $89.7 \%$ of participants were receiving ART. Of those who were not receiving ART, most (85.4\%) were found to be in classes 1 or 2 . Antiretroviral therapy is one of the most important clinical prescriptions for the treatment of HIV, especially in developing countries where the number of PLWH is rising. ${ }^{30}$ It has been recommended that PLWH should accept ART and collaborate with their healthcare providers to increase their chances of success. $^{31,32}$ During ART, PLWH have regular contact with healthcare providers and medical experts to provide various auxiliary services and psychosocial support. ${ }^{33}$ Through contact with ART clinics, PLWH have the opportunity to talk about their problems in counseling sessions, or with other PLWH in their groups. ${ }^{34}$ They also have more opportunities to obtain accurate knowledge and information, which is beneficial for their self- management. In addition, PLWH receiving ART can more easily obtain support from various governmental and nongovernmental organizations, which is critical in living with a chronic disease, ${ }^{35}$ which may enhance the sources of social support and reduce psychological stress and economic burden. PLWH may become active selfmanagers. Therefore, it is necessary to detect HIVpositive people and administer ART as soon as possible. These steps will help PLWH to accept reality, adjust to their own role, adapt psychologically and manage their illness well.

Another main predictor of self-management level is infection route. Our study shows that most $(82.2 \%)$ participants infected through blood/injecting drugs were found to be in classes 1 or 2 . This is consistent with another study in which those infected through blood/ injecting drugs have lower levels of self-management than those with sexually transmitted HIV. ${ }^{36}$ Most (77.63\%) of the PLWH infected through blood/injecting

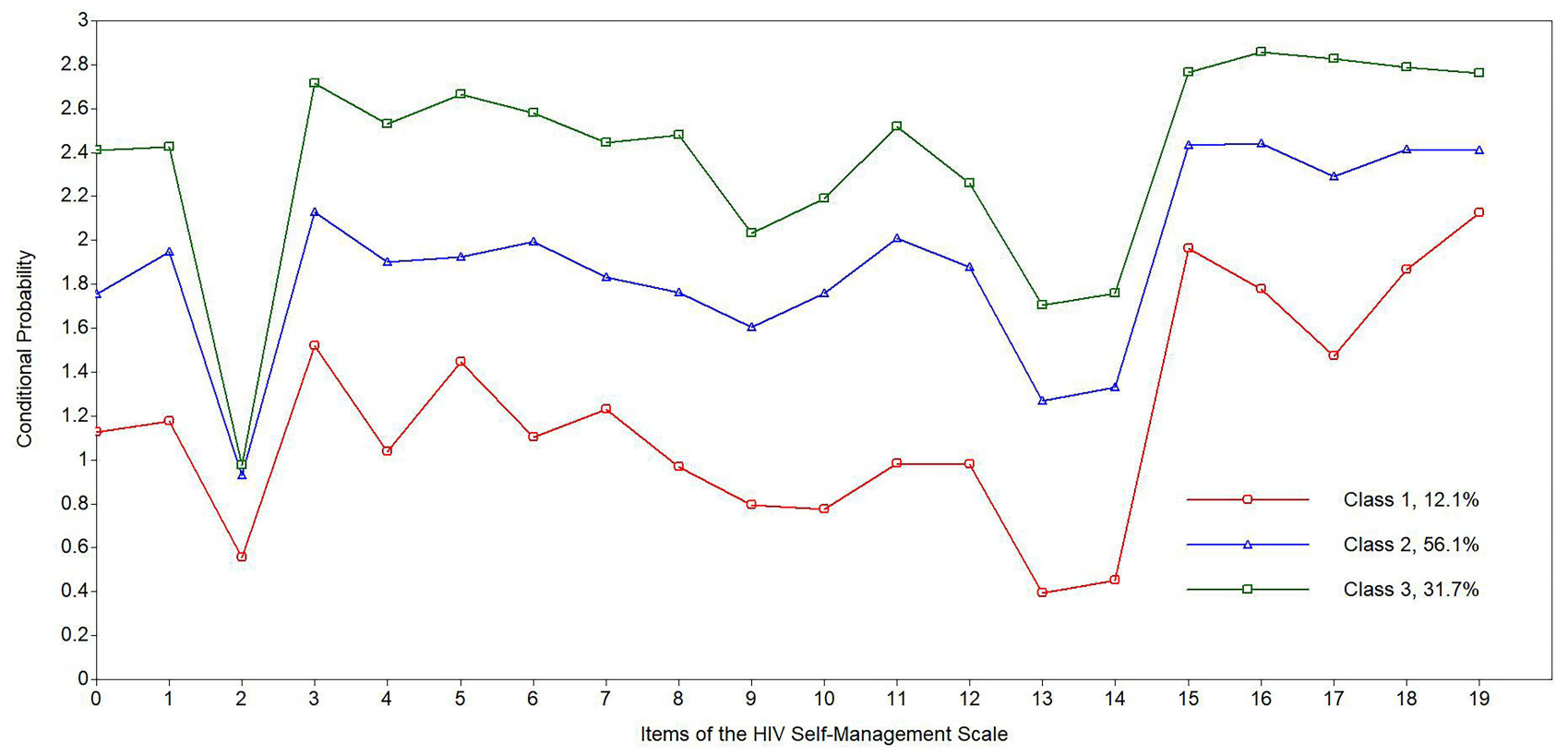

Figure I The conditional probability distribution of the three potential categories of PLWH. 
Table 3 The Distribution of the Sociodemographic and Clinical Data Among the Three Classes $(n=868)$

\begin{tabular}{|c|c|c|c|c|c|}
\hline Variables & Class I (n, \%) & Class 2(n, \%) & Class 3(n, \%) & $\chi^{2}$ & p-value \\
\hline Region & & & & 41.87 & $<0.001$ \\
\hline Chengdu & II (6.10) & $105(58.00)$ & $65(35.90)$ & & \\
\hline Liangshan & $42(14.00)$ & $216(46.60)$ & $64(39.50)$ & & \\
\hline Panzhihua & $51(13.00)$ & $170(67.10)$ & $144(19.90)$ & & \\
\hline Age (years) & & & & 10.23 & 0.037 \\
\hline$\leq 30$ & $13(8.70)$ & $92(61.70)$ & $44(29.50)$ & & \\
\hline $31-50$ & $57(11.60)$ & $290(58.90)$ & $145(29.50)$ & & \\
\hline$>50$ & $34(15.00)$ & $109(48.00)$ & $84(37.00)$ & & \\
\hline Ethnicity & & & & 65.82 & $<0.001$ \\
\hline Minority(Yi people) & $48(10.10)$ & $222(48.70)$ & $46(41.10)$ & & \\
\hline Han nationality & $56(15.20)$ & $269(70.30)$ & $227(14.60)$ & & \\
\hline Educational level & & & & 83.26 & $<0.001$ \\
\hline Illiterate or Primary school & $80(19.00)$ & $26 I(62.10)$ & $79(18.90)$ & & \\
\hline Junior high school & $12(5.80)$ & $99(47.6)$ & $97(46.60)$ & & \\
\hline Senior high school & $4(3.70)$ & $63(58.30)$ & $4 I(38.00)$ & & \\
\hline Junior college or higher & $8(6.10)$ & $68(51.50)$ & $56(42.40)$ & & \\
\hline Religion & & & & 31.41 & $<0.001$ \\
\hline No & $62(11.60)$ & $268(50.10)$ & $205(38.30)$ & & \\
\hline Yes & $42(12.60)$ & $223(67.00)$ & $68(20.40)$ & & \\
\hline \multicolumn{6}{|l|}{ Residence } \\
\hline City/town & $25(6.70)$ & $198(52.80)$ & $152(40.50)$ & 34.54 & $<0.00$ I \\
\hline Countryside & $79(16.00)$ & 293(59.40) & $|2|(24.50)$ & & \\
\hline Occupation & & & & 6.95 & 0.031 \\
\hline Unemployed & $\mathrm{II}(6.80)$ & $90(55.6)$ & $61(37.70)$ & & \\
\hline Employed & $93(13.20)$ & $40 \mathrm{I}(56.80)$ & $212(30.00)$ & & \\
\hline Per capita monthly household income (yuan) & & & & 32.56 & $<0.001$ \\
\hline 0-999 & $66(14.60)$ & $280(61.80)$ & $107(23.60)$ & & \\
\hline $1000-1999$ & $18(13.50)$ & $62(46.60)$ & $53(39.90)$ & & \\
\hline 2000-2999 & $14(8.10)$ & $90(52.00)$ & $69(39.90)$ & & \\
\hline $3000-$ & $6(5.50)$ & $59(54.10)$ & $44(40.40)$ & & \\
\hline Infection route & & & & 29.40 & $<0.001$ \\
\hline Unclear & $38(14.90)$ & $|3|(5 \mid .40)$ & $86(33.70)$ & & \\
\hline Sexual & $36(7.80)$ & $265(57.50)$ & $160(34.70)$ & & \\
\hline Blood/injecting drugs & $30(19.70)$ & $95(62.50)$ & $27(17.80)$ & & \\
\hline ART status & & & & 18.76 & $<0.001$ \\
\hline No & $20(22.50)$ & $56(62.90)$ & $13(14.60)$ & & \\
\hline Yes & $84(10.80)$ & $435(55.80)$ & $260(33.40)$ & & \\
\hline HIV serostatus disclosure & & & & 6.83 & 0.033 \\
\hline No & $15(7.40)$ & $113(55.90)$ & $74(36.60)$ & & \\
\hline Yes & $89(13.40)$ & $378(56.80)$ & $199(29.90)$ & & \\
\hline Gender & & & & 0.78 & 0.679 \\
\hline Male & $68(11.30)$ & $342(57.00)$ & $190(31.70)$ & & \\
\hline Female & $36(13.40)$ & $149(55.60)$ & $83(31.00)$ & & \\
\hline
\end{tabular}

(Continued) 
Table 3 (Continued).

\begin{tabular}{|c|c|c|c|c|c|}
\hline Variables & Class I $(n, \%)$ & Class $2(n, \%)$ & Class 3(n, \%) & $\chi^{2}$ & p-value \\
\hline Marital status & & & & 5.18 & 0.521 \\
\hline Unmarried & $18(9.60)$ & $109(58.30)$ & $60(32.10)$ & & \\
\hline Divorced & $16(12.50)$ & $66(51.60)$ & $46(35.90)$ & & \\
\hline Married & $56(11.80)$ & $273(57.60)$ & $145(30.60)$ & & \\
\hline Widowed & $14(17.70)$ & $43(54.40)$ & $22(27.80)$ & & \\
\hline Years since HIV diagnosis & & & & 8.83 & 0.184 \\
\hline$<1$ & $17(13.50)$ & $70(55.60)$ & $39(31.00)$ & & \\
\hline $1-5$ & $53(12.00)$ & $235(53.30)$ & $153(34.70)$ & & \\
\hline $5.1-10$ & $30(12.10)$ & $156(62.90)$ & $62(25.00)$ & & \\
\hline$>10$ & $4(7.50)$ & $30(56.60)$ & $19(35.80)$ & & \\
\hline
\end{tabular}

Abbreviation: ART, antiretroviral therapy.

drugs who participated in this study were from Liangshan Prefecture. This area is located in the southwestern part of Sichuan Province in a mountainous area, and is an important route of drug entry into Sichuan. Consequently, the injection of drugs is one of the main causes of HIV acquisition in Liangshan Prefecture. ${ }^{37}$ Sharing needles is likely to occur due to a lack of needle hygiene knowledge, difficulty in obtaining needles, and the influence of the Yi culture of sharing. ${ }^{38}$ Use of illicit substances increases the risk of HIV infection, while diminishing both HIV medication adherence and people's self-management of their illness. ${ }^{39}$ Substance abuse behaviors are known to reduce patients' self-control, complicate their ability to selfmanage, and negatively impact the disease outcomes in PLWH. ${ }^{40}$ Therefore, healthcare providers should actively spread knowledge of HIV and safe sexual behavior among drug users to change dangerous behaviors and fundamentally curb the epidemic of HIV.
Due to the varying levels of self-management behavior, the spread of interventions that promote successful selfmanagement is essential for improving health-related quality of life, reducing healthcare costs over time, and preventing the progression of chronic conditions. Mobile health interventions have emerged as a promising tool to support disease self-management among PLWH from all demographic groups and geographic areas. ${ }^{41}$ The use of mobile and web-based technologies in combination is the best solution for the prevention of AIDS. These would be useful in promoting ART and self-management, in providing health information, and in suggesting techniques to help PLWH overcome several of the key barriers to good medication adherence. ${ }^{42}$ We suggest that more relevant studies should be carried out to identify future mobilebased applications for self-management for PLWH.

This is the first reported study to use LCPA to identify different self-management behavior clusters and explore their predictors among a large sample of PLWH. The

Table 4 Predictors of Three Latent Classes of Self-Management Behavior

\begin{tabular}{|l|l|c|c|c|c|}
\hline Models & Predictors & B (SE) & Wald $\left(\chi^{2}\right)$ & p-value & OR (95\% CI) \\
\hline \multirow{2}{*}{$\begin{array}{l}\text { Class 3 versus } \\
\text { Class I }\end{array}$} & Educational level & -1.61 & 6.96 & 0.008 & $0.20(0.06,0.66)$ \\
\cline { 2 - 6 } & Infection route & 0.89 & 7.69 & 0.006 & $2.44(1.30,4.58)$ \\
\cline { 2 - 6 } & ART status & -0.87 & 6.93 & 0.008 & $0.42(0.22,0.80)$ \\
\hline \multirow{2}{*}{$\begin{array}{l}\text { Class 2 versus } \\
\text { Class I }\end{array}$} & Infection route & 1.01 & 7.09 & 0.008 & $2.75(1.31,5.80)$ \\
\cline { 2 - 6 } & ART status & -1.25 & 8.22 & 0.004 & $0.29(0.12,0.67)$ \\
\hline
\end{tabular}

Notes: Class I (a poor level of self-management behavior) was used as the reference group; Class $2=a$ moderate level of self-management behavior; Class $3=a$ good level of self-management behavior. Educational level: I=Illiterate or Primary school, 2=Junior high school, 3=Senior high school, 4= Junior college or higher. Infection route ( $I=$ unclear, $2=$ sexual, $3=$ =blood/injecting drugs). ART status ( $(=\mathrm{No}, 2=$ Yes)

Abbreviations: OR, odds ratio; $\mathrm{Cl}$, confidence interval; ART, antiretroviral therapy. 
findings show the pertinence of this approach to current and future nursing research. The study has the potential to assist healthcare professionals in quickly identifying PLWH who are at a high risk of low levels of self-management, based on their individual characteristics. The approach is also highly valuable in clinical nursing practice, as it provides a clear focus for healthcare providers to begin to develop targeted interventions designed to promote the uptake of healthy behavior for specific subgroups.

There are several limitations in this study. First, this study provided a preliminarily exploration of self-management behavior clusters in PLWH and identified some target predictive factors. However, we could not determine the exact causality between self-management and predictors due to the cross-sectional design. Therefore, there is a need for continuing longitudinal research and multicenter studies to confirm our findings and explore its causality. Second, the generalizability of the findings is potentially limited by the fact that all the respondents were from Sichuan Province in China, so the results cannot be generalized to all PLWH. Future studies should be conducted in the form of multicenter surveys elsewhere in China and the world. Third, a limited number of contextual risk and protective factors in the IFSMT were included in the study. Other influencing factors in the "process of self-management" (eg, self-efficacy, self-regulation abilities, and social facilitation) should be explored in future studies.

\section{Conclusion}

The findings indicated that the level of self-management behaviors among PLWH in China is inadequate. Levels of self-management were divided into three latent categories: Class 1 (a poor level of self-management), Class 2 (a moderate level of self-management), and Class 3 (a good level of self-management). Most (68.2\%) participants showed a poor or moderate level of self-management. ART status, infection route, and educational level were the main predictors of participants' self-management behavior. Those with a lower educational level, who were infected through blood/injecting drugs, or who were not receiving ART had a significantly lower level of selfmanagement. These results could help healthcare professionals to quickly identify PLWH who are at a high risk for low levels of self-management, using individual characteristics, as well as provide a scientific basis for the development of effective and targeted programs to improve self-management levels in PLWH.

\section{Acknowledgments}

The authors gratefully appreciate the support of the investigators and all the participants.

\section{Author Contributions}

All authors made a significant contribution to the work reported, whether that is in the conception, study design, execution, acquisition of data, analysis and interpretation, or in all these areas; took part in drafting, revising or critically reviewing the article; gave final approval of the version to be published; have agreed on the journal to which the article has been submitted; and agree to be accountable for all aspects of the work.

\section{Funding}

The work was supported by the Sichuan Provincial Project for Major Diseases Prevention and Control (Registration number: 70, 2017).

\section{Disclosure}

The authors have no conflicts of interest to declare.

\section{References}

1. Zhang P, Gao J, Wang Y, Sun Q, Sun X. Effect of chronic disease self-management program on the quality of life of HIV-infected men who have sex with men: an empirical study in Shanghai, China. Int J Health Plann Manage. 2019;34(3):1055-1064. doi:10.1002/ hpm. 2874

2. UNAIDS. UNAIDS data 2018. Available from: http://www.unaids.org/ en/resources/documents/2018/unaids-data-2018. Accessed July 26, 2018.

3. NCAIDS, NCSTD, China CDC. Update on the AIDS/STD epidemic in China in December, 2016. Chin J AIDS STD. 2017;23(02):93. doi:10.13419/j.cnki.aids.2017.02.01

4. Siddiqi AE, Hall HI, Hu X, Song R. Population-Based Estimates of Life Expectancy After HIV Diagnosis: united States 2008-2011. J Acquir Immune Defic Syndr. 2016;72(2):230-236. doi:10.1097/ QAI.0000000000000960

5. Webel AR, Higgins PA. The relationship between social roles and self-management behavior in women living with HIV/AIDS. Womens Health Issues. 2012;22(1):e27-e33. doi:10.1016/j.whi.2011.05.010

6. Rotheram-Borus MJ, Ingram BL, Swendeman D, Lee A. Adoption of self-management interventions for prevention and care. Prim Care. 2012;39(4):649-660. doi:10.1016/j.pop.2012.08.006

7. Zhu Y, Liu J, Qu B, Hu B, Zhang Y. Relationship between quality of life and unprotected anal intercourse among Chinese men who have sex with men: a cross-sectional study. BMC Public Health. 2016;16:382. doi:10.1186/s12889-016-3076-Z

8. Areri HA, Marshall A, Harvey G. Interventions to improve self-management of adults living with HIV on Antiretroviral Therapy: a systematic review. PLoS One. 2020;15(5):e232709. doi:10.1371/journal.pone.0232709

9. Allegrante JP, Wells MT, Peterson JC. Interventions to Support Behavioral Self-Management of Chronic Diseases. Annu Rev Public Health. 2019;40:127-146. doi:10.1146/annurev-publhealth-040218044008 
10. White House Office of National AIDS Policy. The National HIV/ AIDS Strategy for the United States: updatedto 2020. Available from: https://www.hiv.gov/federal-response/hiv-national-strategic-plan /national-hiv-aids-strategies-2010-2020. Accessed July 30, 2015.

11. Russell S, Martin F, Zalwango F, et al. Finding Meaning: HIV Self-Management and Wellbeing among People Taking Antiretroviral Therapy in Uganda. PLoS One. 2016;11(1):e0147896. doi:10.1371/journal.pone.0147896

12. Schulman-Green D, Jaser S, Martin F, et al. Processes of self-management in chronic illness. J Nurs Scholarsh. 2012;44 (2):136-144. doi:10.1111/j.1547-5069.2012.01444.x

13. Kathleen J. Definitions, frameworks, and theoretical issues in self-management. J Pediatr Rehabil Med. 2017;10(3-4):169-176. doi:10.3233/PRM-170461

14. Ryan P, Sawin KJ. The Individual and Family Self-Management Theory: background and perspectives on context, process, and outcomes. Nurs Outlook. 2009;57(4):217-225.e6. doi:10.1016/j. outlook.2008.10.004

15. Areri H, Marshall A, Harvey G. Factors influencing self-management of adults living with HIV on antiretroviral therapy in Northwest Ethiopia: a cross-sectional study. BMC Infect Dis. 2020;20(1):879. doi:10.1186/s12879-020-05618-y

16. Webel AR, Cuca Y, Okonsky JG, Asher AK, Kaihura A, Salata RA. The impact of social context on self-management in women living with HIV. Soc Sci Med. 2013;87:147-154. doi:10.1016/j. socscimed.2013.03.037

17. Wang H, Chen AC, Wan S, Chen H. Status and associated factors of self-management in people living with HIV/AIDS in Liangshan area, China: a cross-sectional study. Patient Prefer Adherence. 2019;13:863-870. doi:10.2147/PPA.S203799

18. Kim GS, Chu SH, Park Y, et al. Psychometric Properties of the Korean Version of the HIV Self-Management Scale in Patients with HIV. J Korean Acad Nurs. 2015;45(3):439-448. doi:10.4040/ jkan.2015.45.3.439

19. Han Y, Xiao SW, Chen H. Influencing Factors and Present Status of Self-management Among People Living With HIV/AIDS. J Prevention Med Information. 2019;35(4):366-371.

20. Wang HH, Gao C, Zhang L, Chen C, Xiao XL. Study on the status of disease self-management in People Living With HIV in Hunan province and its influencing factors. Chine Nursing Management. 2019;19(12):1779-1785. doi:10.3969/j.issn.1672-1756.2019.12.005

21. Dong K, Ye L, Leng Y, et al. Prevalence of HIV-1 Drug Resistance among Patients with Antiretroviral Therapy Failure in Sichuan, China, 2010-2016. Tohoku J Exp Med. 2019;247(1):1-12. doi:10.1620/tjem.247.1

22. Nylund-Gibson K, Choi AY. Ten frequently asked questions about latent class analysis. Transl Issues Psychol Sci. 2018;4(4):440-461. doi: $10.1037 /$ tps 0000176

23. Webel AR, Asher A, Cuca Y, et al. Measuring HIV self-management in women living with HIV/AIDS: a psychometric evaluation study of the HIV Self-management Scale. J Acquir Immune Defic Syndr. 2012;60(3):e72-e81. doi:10.1097/QAI.0b013e318256623d

24. McArdle JJ. Latent variable modeling of differences and changes with longitudinal data. Annu Rev Psychol. 2009;60:577-605. doi:10.1146/annurev.psych.60.110707.163612

25. Park J, Moser DK, Griffith K, Harring JR, Johantgen M. Exploring Symptom Clusters in People With Heart Failure. Clin Nurs Res. 2019;28(2):165-181. doi:10.1177/1054773817729606

26. He J, Chen X, Fan X, Cai Z, Hao S. Profiles of parent and peer attachments of adolescents and associations with psychological outcomes. Child Youth Serv Rev. 2018;94:163-172. doi:10.1016/j. childyouth.2018.10.001

27. Vrieze SI. Model selection and psychological theory: a discussion of the differences between the Akaike information criterion (AIC) and the Bayesian information criterion (BIC). Psychol Methods. 2012;17 (2):228-243. doi:10.1037/a0027127
28. Solomon P, Salbach NM, O'Brien KK, Nixon S, Baxter L, Gervais N. Evaluation of a Community-Based Self-Management Program to Increase Access to Rehabilitation for People Living with HIV. $J$ Int Assoc Provid AIDS Care. 2019;18:2325958219883334. doi: $10.1177 / 2325958219883334$

29. Efendi F, Pratama ER, Hadisuyatmana S, Indarwati R, Lindayani L, Bushy A. HIV-related knowledge level among Indonesian women between 15 years and 49 years of age. Afr Health Sci. 2020;20 (1):83-90. doi:10.4314/ahs.v20i1.13

30. Nhavoto JA, Grönlund A, Klein GO. Mobile health treatment support intervention for HIV and tuberculosis in Mozambique: perspectives of patients and healthcare workers. PLoS One. 2017;12(4):e0176051. doi:10.1371/journal.pone.0176051

31. Millard T, Elliott J, Girdler S. Self-management education programs for people living with HIV/AIDS: a systematic review. AIDS Patient Care STDS. 2013;27(2):103-113. doi:10.1089/apc.2012.0294

32. Swendeman D, Ramanathan N, Baetscher L, et al. Smartphone self-monitoring to support self-management among people living with HIV: perceived benefits and theory of change from a mixed-methods randomized pilot study. J Acquir Immune Defic Syndr. 2015;69:S80-S91. doi:10.1097/QAI.0000000000000570

33. Chandran A, Benning L, Musci RJ, et al. The Longitudinal Association between Social Support on HIV Medication Adherence and Healthcare Utilization in the Women's Interagency HIV Study. AIDS Behav. 2019;23(8):2014-2024. doi:10.1007/s10461-018-2308$\mathrm{x}$

34. Martin F, Russell S, Seeley J. Higher quality of life and lower depression for people on ART in Uganda as compared to a community control group. PLoS One. 2014;9(8):e105154. doi:10.1371/journal.pone.0105154

35. Wang D, Mei G, Xu X, et al. Chinese non-governmental organizations involved in HIV/AIDS prevention and control: intra-organizational social capital as a new analytical perspective. Biosci Trends. 2016;10(5):418-423. doi:10.5582/bst.2016.01134

36. Wang $H$, Yang $H$, Chen $H$. Status and influencing factors of self-management for people living with HIV/AIDS in the areas with different HIV/AIDS epidemics in Liangshan. Chine $J$ AIDS \& STD. 2019;25(2):140-143+157.

37. Zhai WW, Yang SJ, Yu G, et al. Analysis of the HIV infection and its influencing factors in drug addicts in Liangshan Prefecture of Sichuan Province between 2009 and 2015. Modern Preventive Medicine. 2017;44(18):3265-3269.

38. Yang SJ, Zhai WW, Ji-K CN, et al. Risk factors of HIV infection among Yi people and other ethnic groups based on the HIV/AIDS sentinel surveillance in the three counties of Liangshan. Chine $J$ AIDS \& STD. 2018;24(03):257-260.

39. Zhang Y, Wilson TE, Adedimeji A, et al. The Impact of Substance Use on Adherence to Antiretroviral Therapy Among HIV-Infected Women in the United States. AIDS Behav. 2018;22(3):896-908. doi:10.1007/s10461-017-1808-4

40. Brion JM, Rose CD, Nicholas PK, et al. Unhealthy substance-use behaviors as symptom-related self-care in persons with HIV/AIDS. Nurs Health Sci. 2011;13(1):16-26. doi:10.1111/j.14422018.2010.00572.x

41. Ernsting C, Dombrowski SU, Oedekoven M, et al. Using Smartphones and Health Apps to Change and Manage Health Behaviors: a Population-Based Survey. J Med Internet Res. 2017;19 (4):e101. doi:10.2196/jmir.6838

42. Niakan S, Mehraeen E, Noori T, Gozali E. Web and Mobile Based HIV Prevention and Intervention Programs Pros and Cons - A Review. Stud Health Technol Inform. 2017;236:319-327. doi:10.3233/978-1-61499-759-7-319 


\section{Publish your work in this journal}

Patient Preference and Adherence is an international, peer-reviewed, open access journal that focusing on the growing importance of patient preference and adherence throughout the therapeutic continuum. Patient satisfaction, acceptability, quality of life, compliance, persistence and their role in developing new therapeutic modalities and compounds to optimize clinical outcomes for existing disease

states are major areas of interest for the journal. This journal has been accepted for indexing on PubMed Central. The manuscript management system is completely online and includes a very quick and fair peer-review system, which is all easy to use. Visit http:// www.dovepress.com/testimonials.php to read real quotes from published authors. 